\title{
Silencing BMI1 radiosensitizes human breast cancer cells by inducing DNA damage and autophagy
}

\author{
JAMES GRIFFITH $^{1}$, DANIEL ANDRADE ${ }^{1}$, MEGHNA MEHTA ${ }^{1}$, WILLIAM BERRY ${ }^{2}$, \\ DORIS M. BENBROOK ${ }^{3,5}$, NATARAJAN ARAVINDAN ${ }^{1,5}$, TERENCE S. HERMAN ${ }^{1,5}$, \\ RAJAGOPAL RAMESH ${ }^{4,5}$ and ANUPAMA MUNSHI ${ }^{1,5}$ \\ Departments of ${ }^{1}$ Radiation Oncology, ${ }^{2}$ Cell Biology, ${ }^{3}$ Obstetrics and Gynecology, and \\ ${ }^{4}$ Pathology, University of Oklahoma Health Sciences Center; ${ }^{5}$ Stephenson Cancer Center, \\ University of Oklahoma Health Sciences Center, Oklahoma City, OK 73104, USA
}

Received September 7, 2016; Accepted January 25, 2017

DOI: $10.3892 /$ or.2017.5478

\begin{abstract}
Overexpression of BMI1 in human cancer cells, a member of the polycomb group of repressive complexes, correlates with advanced stage of disease, aggressive clinicopathological behavior, poor prognosis, and resistance to radiation and chemotherapy. Studies have shown that experimental reduction of BMI1 protein level in tumor cells results in inhibition of cell proliferation, induction of apoptosis and/or senescence, and increased susceptibility to cytotoxic agents and radiation therapy. Although a role for BMI1 in cancer progression and its importance as a molecular target for cancer therapy has been established, information on the impact of silencing BMI1 in triple-negative breast cancer (TNBC) and its consequence on radiotherapy have not been well studied. Therefore, in the present study we investigated the potential therapeutic benefit of radiation therapy in BMI1-silenced breast cancer cells and studied the mechanism(s) of radiosensitization. Human MDA-MB-231 and SUM159PT breast cancer cells that were either stably transfected with a lentiviral vector expressing BMI1 shRNA (shBMI1) or control shRNA (shControl) or transient transfection with a BMI1-specific siRNA were used. Silencing of BMI1 resulted in marked reduction in BMI1 both at the mRNA and protein level that was accompanied by a significant reduction in cell migration compared to control cells. Further, BMI1 knockdown produced a marked enhancement of DNA damage as evidenced by Comet Assay and $\gamma \mathrm{H} 2 \mathrm{AX}$ foci, resulting in a dose-dependent radiosensitization effect. Molecular studies revealed modulation of protein expression that is associated with the DNA damage response (DDR) and
\end{abstract}

Correspondence to: Dr Anupama Munshi, Department of Radiation Oncology, Stanton L. Young Biomedical Research Center, Suite \#1403, University of Oklahoma Health Sciences Center, 975 NE 10th Street, Oklahoma City, OK 73104, USA

E-mail: anupama-munshi@ouhsc.edu

Key words: autophagy, BMI1, breast cancer, radiation, DNA damage autophagy pathways. Our results demonstrate that BMI1 is an important therapeutic target in breast cancer and suppression of BMI1 produces radiation sensitivity. Further, combining BMI1targeted therapeutics with radiation might benefit patients diagnosed with TNBC.

\section{Introduction}

The polycomb group $(\mathrm{PcG})$ of transcription factor proteins form transcriptional repressor modules that play crucial roles in many physiological processes, including cell differentiation, stem cell self-renewal, and gene silencing through histone modifications (1). Numerous studies have shown that PcG proteins are involved in malignant transformation and tumor development in various cancer types (2). B cellspecific Moloney murine leukemia virus integration site 1 (BMI1), a member of the PcG complex, plays an essential role in the maintenance and self-renewal of hematopoietic and neural stem cells, at least partly by silencing the Ink4a/Arf locus $(3,4)$. BMI1 has also been linked with a multitude of cellular processes, including cell cycle progression, apoptosis, epithelial-to-mesenchymal transition (EMT), senescence, immortalization and/or induction of telomerase (5-7). BMI1 overexpression is associated with disease progression and poor clinical outcome in a number of human malignancies (8-11). Although BMI1 plays a critical role in cancer, the precise molecular mechanism by which it contributes to cancer development and therapy failure remains poorly understood.

Several independent studies have demonstrated that genetic silencing and pharmacologic inhibition of BMI1 suppresses the growth of various cancers, induces cell cycle arrest, apoptosis and senescence, and increases susceptibility to chemotherapeutic agents and ionizing radiation (12-14). In normal human keratinocytes, BMI1 elicits radioprotective effects by mitigating the genotoxic effects of ionizing radiation (IR) (15). In nasopharyngeal carcinoma cells, targeting BMI1 expression increases their susceptibility to radiation through the induction of oxidative stress and apoptosis (13). Elevated expression of BMI1 has been shown to radioprotect CD133-positive cancer-initiating neural stem cells through recruitment of DNA damage response (DDR) machinery to 
DSBs after exposure to radiation (16). Although a role for BMI1 in cancer progression and its importance as a target for therapy has been reported, its role in radiosensitization of breast cancer has not been investigated.

In the present study, we demonstrate that silencing BMI1 sensitizes MDA-MB-231 and SUM159PT breast cancer cells to ionizing radiation. We also show that this sensitization occurs through induction of both the DDR and autophagy pathways. These results indicate that BMI1 may play an important role in radioresistance, and that BMI1 suppression may be an important therapeutic target for breast cancer.

\section{Materials and methods}

Cell lines. Human MDA-MB-231 breast cancer cell line obtained from American Type Culture Collection (ATCC; Manassas, VA, USA) was maintained in $\alpha$-MEM (Cellgro, Manassas, VA, USA) containing $10 \%$ fetal bovine serum, $2 \mathrm{mmol} / \mathrm{l} \mathrm{L}$-glutamine, and $2 \mathrm{mmol} / 1$ penicillin-streptomycin. SUM159PT cells were obtained from Asterand Bioscience (Detroit, MI, USA) and maintained in Ham's F-12 media supplemented with $5 \%$ heat-inactivated FBS, 2 mmol/1 penicillin-streptomycin, $10 \mathrm{mM}$ Hepes, and $1 \mu \mathrm{g} / \mathrm{ml}$ insulin. All cultures were maintained at $37^{\circ} \mathrm{C}$ in an atmosphere of $5 \%$ $\mathrm{CO}_{2}$ and $95 \%$ room air.

Plasmid construction. Sequences (miR shControl: Sense 5'-AGCGATCTCGCTTGGGCGAGAGTAAGTATGAAG CCACAGATGTGACTTACTCTCGCCCAACGAGAG-3', Antisense 5'-GGCAACTCTCGCTTGGGCGAGAGTAA GTACATCTGTGGCTTCACTACTTACTCTCGCCCAAG CGAGAT-3'; miR shBMI1: Sense 5'-AGCGATCCAAGAT ATTGTATACAAATTAGTGAAGCCACAGATGTAATT TGTATACAATATCTTGGAG-3', Antisense 5'-GGCACTC CAAGATATTGTATACAAATTACATCTGTGGCTTCAC TAATTTGTATACAATATCTTGGAT-3') were cloned into pEN_RmiRc2 (17). Then, entry vectors containing shRNA sequence were recombined with the lentiviral destination vector CMV PURO DEST according to manufacturer's recommendations (Invitrogen, Grand Island, NY, USA).

siRNA transfection. Transfection of SUM159PT cells with human BMI1 siRNA (siBMI1) and non-targeting siRNA\#3 (siScr) (GE Dharmacon, Lafayette, CO, USA) was performed in 60-mm dishes using DharmaFECT 2 transfection reagent (GE Dharmacon) according to manufacturer's instructions. Cells were transfected with siRNA $(20 \mathrm{nM})$ in serum-free medium. Six hours after transfection, the media was replaced with fresh medium containing $2 \%$ serum. The next day the cells were irradiated (5 Gy) and harvested after specified incubation periods for further experiments.

Wound healing assay. MDA-MB-231 shControl and shBMI1 cells were seeded in 60-mm dishes and grown to $80-85 \%$ confluence. After $24 \mathrm{~h}$ of incubation the cell monolayers were wounded longitudinally using a $200 \mu$ l pipette tip. Cells were washed once to remove detached and injured cells and were visually assessed immediately, with live images captured by light microscopy using an attached CCD camera. After an additional 24 and $48 \mathrm{~h}$ incubation, cells were stained with crystal violet and pictures were taken at the same location as the initial image to determine any defects in cell migration. Experiments were done in triplicate. Three random fields of each well were recorded.

Cell migration assay. Cells were seeded in the upper chamber of the Transwell ( $8 \mu \mathrm{m}$; BD Biosciences, Bedford, MA, USA) in medium containing $2 \% \mathrm{FBS}$ and placed in a 6 -well plate filled with $2 \mathrm{ml}$ of medium containing 20\% FBS (lower chamber). After 24, 48, and $72 \mathrm{~h}$ incubation, the inserts were removed, processed, and the number of migrated cells counted as previously described (18).

Clonogenic survival. The effectiveness of BMI1 knockdown (in both shBMI1 and siBMI1-treated cells) and ionizing radiation was assessed with clonogenic assays as previously described (19,20). Cells were irradiated with a high dose-rate ${ }^{137}$ Cesium (Cs) unit at room temperature. After treatment, known number of cells were re-plated in triplicate in $60-\mathrm{mm}$ tissue culture dishes and returned to the incubator to allow macroscopic colony development. Ten-to-twelve days after seeding, colonies were stained with $0.5 \%$ gentian violet in methanol. The number of colonies formed in each treatment group was counted, with a cutoff of 50 viable cells per colony. The percent plating efficiency (PE) and fraction surviving a given treatment was calculated based on the survival of nonirradiated control or BMI1 knockdown cells. Survival curves were generated after normalizing for the cytotoxicity generated by control alone.

Western blot analysis. Protein extracts, separated by SDS-PAGE and transferred onto PVDF membranes, were probed with antibodies against LC3B, p62 (Santa Cruz Biotechnology, Dallas, TX, USA), BMI1, p38, phospho-p38, Akt, phospho-Akt, Bcl2, Mcl1, MRE11, Bax, DNA-PK, Ku70, Ku80 (Cell Signaling Technology, Beverly, MA, USA), and ATM (Gene Tex, Irvine, CA, USA). $\beta$-actin was used as an internal loading control (Santa Cruz Biotechnology). Proteins were detected using the appropriate HRP-conjugated secondary antibodies and visualized by enhanced chemiluminescence with ECL Plus reagent (Amersham Pharmacia Biotech, Arlington Heights, IL, USA).

Quantitative polymerase chain reaction. Total RNA was isolated using TRIzol (Life Technologies, Grand Island, NY, USA) and subjected to reverse transcription using the Omniscript RT kit (Qiagen Inc., Hercules, CA, USA). Obtained cDNA was used to perform real-time (RT)-PCR (Bio-Rad CFX96 ${ }^{\mathrm{TM}}$ Touch Real-time PCR Detection System) with PerfeCTa SYBR Green Fast Mix (Quanta BioSciences, Gaithersburg, MD, USA). The primers for human BMI1, Atg3, Atg5, Atg7, Atg12, LC3A, LC3B, and GAPDH were obtained from IDT Inc. (Coralville, IA, USA). The comparative Ct (cycle threshold) method was used to calculate the relative abundance of mRNA compared with that of GAPDH expression in triplicate experiments.

Immunofluorescent staining for $\gamma$-H2AX. Cells were grown for $24 \mathrm{~h}$ on coverslips. At specified times, cells were treated with 2 Gray (Gy) radiation, fixed and stained as previously described $(19,20)$. Stained slides were imaged using a Nikon 
A

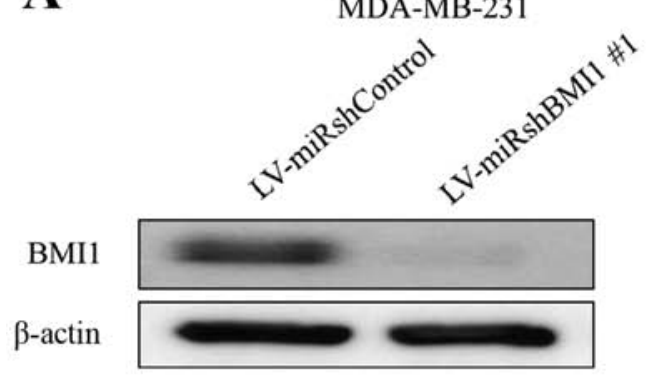

B

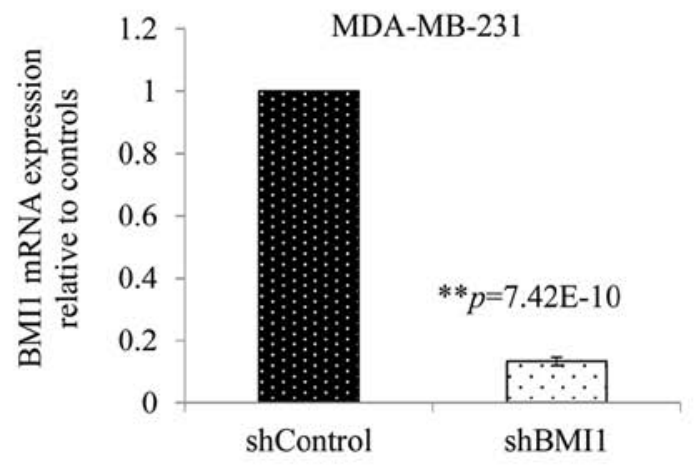

C

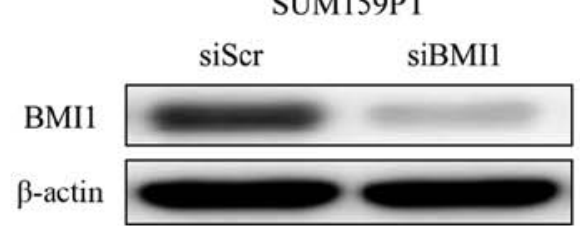

D

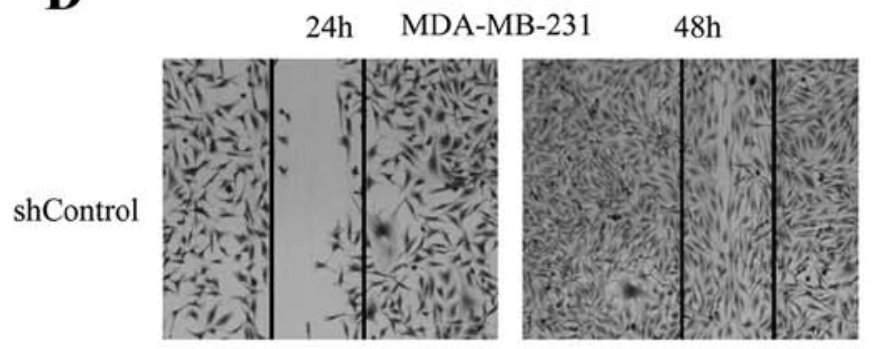

shBMI1
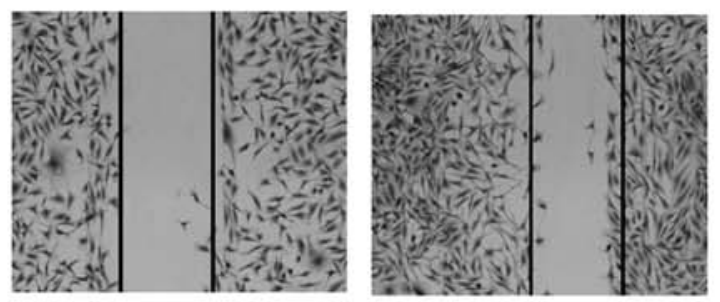

$\mathbf{E}$

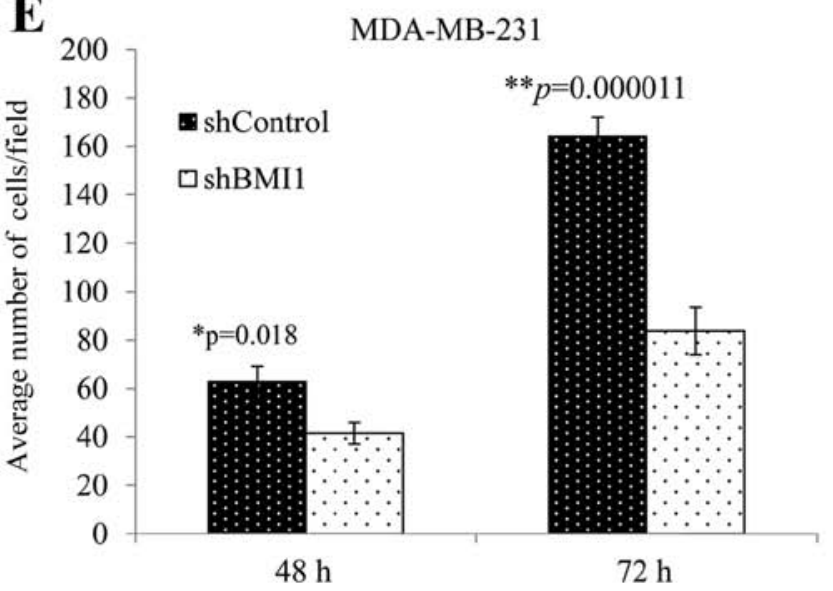

Figure 1. BMI1 knockdown evaluated by (A) western blotting and (B) quantitative RT-PCR in MDA-MB-231 cells. (C) BMI1 expression in siBMI1-treated SUM159PT cells. (D) wound healing assay in MDA-MB-231 cells showed shBMI1 reduced cell migration and (E) tumor cell migration of MDA-MB-231 cells was significantly inhibited by silencing BMI1. Error bars represent standard error of the mean.

fluorescence microscope with an attached CCD camera using NIS-Elements imaging software (Nikon Instruments, Melville, NY, USA). For each treatment, $\gamma-\mathrm{H} 2 \mathrm{AX}$ foci were counted in at least 50 cells from the stored images.

Comet assay. DSBs were detected using a Comet Assay kit (Trevigen, Gaithersburg, MA, USA) according to the manufacturer's instructions. Briefly, cells were grown on 35-mm dishes. Twenty-four hours later, cells were irradiated at $20 \mathrm{~Gy}$, harvested at specified time points, washed twice, resuspended at $1 \times 10^{5}$ cells $/ \mathrm{ml}$ in ice-cold $1 \mathrm{X}$ PBS, combined with low-melting (LM) agarose at a ratio of 1:10 (v/v) and spread on the Comet slide. The slides were allowed to solidify for $30 \mathrm{~min}$ in the dark at $4^{\circ} \mathrm{C}$ and then submerged in precooled, neutral lysis buffer at $4^{\circ} \mathrm{C}$ overnight. After lysis, slides were washed in $1 \mathrm{X}$ TBE buffer $[0.89 \mathrm{~mol} / 1$ Tris, $0.88 \mathrm{~mol} / 1$ boric acid, $2 \mathrm{mmol} / 1$ EDTA (pH 8.3)] for $15 \mathrm{~min}$ and subjected to electrophoresis at $1.0 \mathrm{~V} / \mathrm{cm}$ for $45 \mathrm{~min}$. The slides were then rinsed with distilled water, placed in $70 \%$ ethanol for $5 \mathrm{~min}$, dried at $37^{\circ} \mathrm{C}$ for $15 \mathrm{~min}$ and stained with SYBR Green for $30 \mathrm{~min}$ in the dark. Comet images were obtained using a Nikon fluorescence microscope with an attached CCD camera and NIS-Elements imaging software (Nikon Instruments) and analyzed using Casplab comet assay software. The Olive tail moment was determined for 50 cells in each sample.

Statistical analysis. Data analysis was performed using the paired t-test. Data are presented as the mean \pm standard deviation at least for three independent experiments. $\mathrm{p}<0.05$ was considered to indicate a statistically significant difference.

\section{Results}

BMI1 downregulation reduces the migration potential of MDA-MB-231 cells. MDA-MB-231 cells transduced with a vector expressing an shRNA against human BMI1 (shBMI1 cells) demonstrated greatly reduced BMI1 protein and mRNA expression compared with those transduced with a vector control (shControl cells) (Fig. 1A and B). Similarly, siBMI1 reduced BMI1 protein levels significantly in SUM159PT cells when compared with non-targeted siRNA (siScr) (Fig. 1C). 
A



B

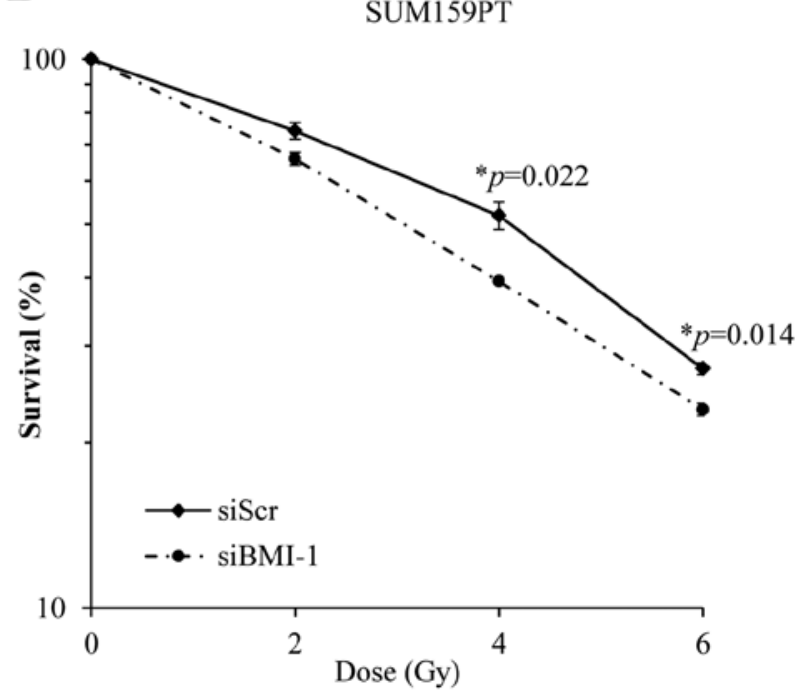

Figure 2. Silencing BMI1 radiosensitizes TNBC cells. (A) MDA-MB-231 shControl or shBMI1 cells and (B) SUM159PT cells treated with siScr or siBMI1 were assessed for radiosensitization by clonogenic cell survival assay immediately after irradiation. BMI1 silencing radiosensitized both cell lines compared to controls. The results represent the average of three independent experiments, each plated in triplicate. Error bars represent standard error of the mean.

To investigate whether BMI1 knockdown affects cellular migration, we performed the wound healing and Transwell assays. The wound healing assay showed that inhibition of BMI1 dramatically impaired the wound healing rate in MDA-MB-231 cells as observed in their ability to fill the gap at 24 and $48 \mathrm{~h}$ (Fig. 1D). The Transwell assay showed a significant reduction in the migration potential in shBMI1 cells with average cells per field decreasing by $34 \%$, and $49 \%$ compared with shControl cells at 48 and $72 \mathrm{~h}$, respectively (Fig. 1E; $\mathrm{p}<0.02$ ). These two assays showed that inhibition of BMI1 impaired cell migration in MDA-MB-231, a very important hallmark in cancer progression.

Knockdown of BMI1 sensitizes breast cancer cells to radiation and amplifies DNA damage and prolongs repair. We investigated the effect of silencing BMI1 on the radiosensitivity of MDA-MB231 and SUM159PT cells by assessing their clonogenic potential. BMI1 knockdown was achieved in MDA-MB-231 cells using an shRNA against BMI1 (as described above) and in SUM159PT cells using a BMI1-specific siRNA. We found that silencing BMI1 suppressed the clonogenic survival and radiosensitized MDA-MB-231 cells (Fig. 2A). Survival at 2 Gy (SF2) was reduced from $54 \pm 2 \%$ in the shControl cells to $45 \pm 1 \%$ in shBMI1 $(\mathrm{p}=0.01)$ cells. Dose enhancement factor was then calculated by dividing the radiation dose that produced $10 \%$ cell survival on the radiation-only survival curve by that for the corresponding shBMI1 plus ionizing radiation curve. The dose enhancement factor for MDA-MB-231 cells was 1.12.

Similar results were obtained using the SUM159PT cell line following treatment with siBMI1. Treatment with siBMI1 resulted in an increase in the radiosensitivity of SUM159PT cell line as compared with siScr (Fig. 2B). Exposure of SUM159PT cells to siBMI1 reduced the surviving fraction at 2 Gy from $74 \pm 2.5 \%$ in the siScr to $65.8 \pm 1.7 \%$ in siBMI1-treated cells $(\mathrm{p}=0.08)$. The dose enhancement factor for SUM159PT cells, calculated at $50 \%$ survival, was 1.36 .
We also assessed the involvement of the DDR in shBMI1 radiosensitization by determining the number of $\gamma-\mathrm{H} 2 \mathrm{AX}$ foci, an indicator of radiation-induced DSBs. shControl and shBMI1 cells were treated with radiation (2Gy) and immunostained at 4 and $24 \mathrm{~h}$ post-radiation. $\gamma-\mathrm{H} 2 \mathrm{AX}$ foci could be clearly distinguished after irradiation ( $2 \mathrm{~Gy}$ ) as shown by the micrographs in Fig. 3A. The average number of $\gamma$-H2AX foci per cell were counted and the results are presented in Fig. 3B. At $0 \mathrm{~h}$ (no irradiation), the average number of $\gamma-\mathrm{H} 2 \mathrm{AX}$ foci per cell was significantly greater in the BMI1-silenced population than in the shControl $(\mathrm{p}=0.005$; Fig. 3B). After irradiation with $2 \mathrm{~Gy}$, the average number of $\gamma$-H2AX foci in shBMI1 cells significantly increased compared to the shControl-radiation group at $4 \mathrm{~h}(\mathrm{p}=0.022)$ and $24 \mathrm{~h}(\mathrm{p}=0.00018$; Fig. 3B). An increase in $\gamma$-H2AX levels in the shBMI1 plus radiation group was also observed by western blot analysis (Fig. 3C). The sustained increase in $\gamma-\mathrm{H} 2 \mathrm{AX}$ foci after radiation in BMI1-silenced cells suggests that shBMI1-mediated radiosensitization involves an enhancement of the DDR and inhibition of DNA repair.

To further understand the extent of BMI1's influence on DSBs, we performed the neutral Comet assay. This technique allows the quantification of DSBs by measuring the length and area of the 'comet' represented by the 'Olive tail moment' (OTM). A greater tail length and a greater tail area indicate that more DNA breaks have occurred. Elevated basal levels of DNA damage (no radiation) were observed in shBMI1 cells compared with the shControl cells (Fig. 4A and B). Combined shBMI1 plus radiation (20 Gy), however, resulted in greater DNA damage at $24 \mathrm{~h}$ after radiation (Fig. 4B; p<0.001), suggesting that the repair of radiation-induced DNA damage can be significantly suppressed upon BMI1 inhibition in MDA-MB-231 cells.

BMII modulates expression of DDR and the Akt and $M A P K$ pathways. In view of the increased DNA damage 
A

MDA-MB-231

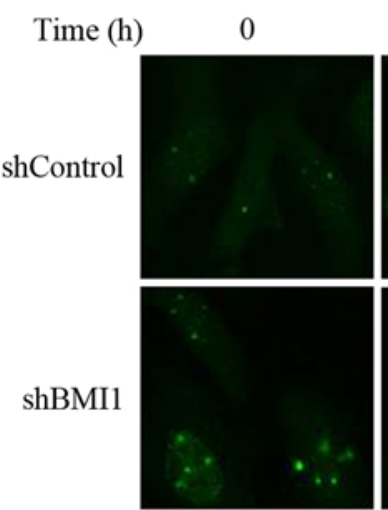

4

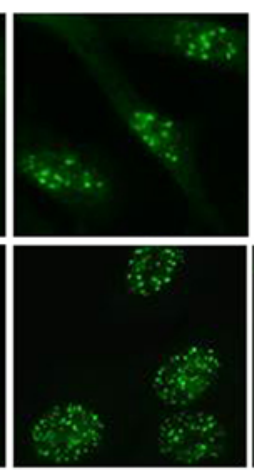

24



B
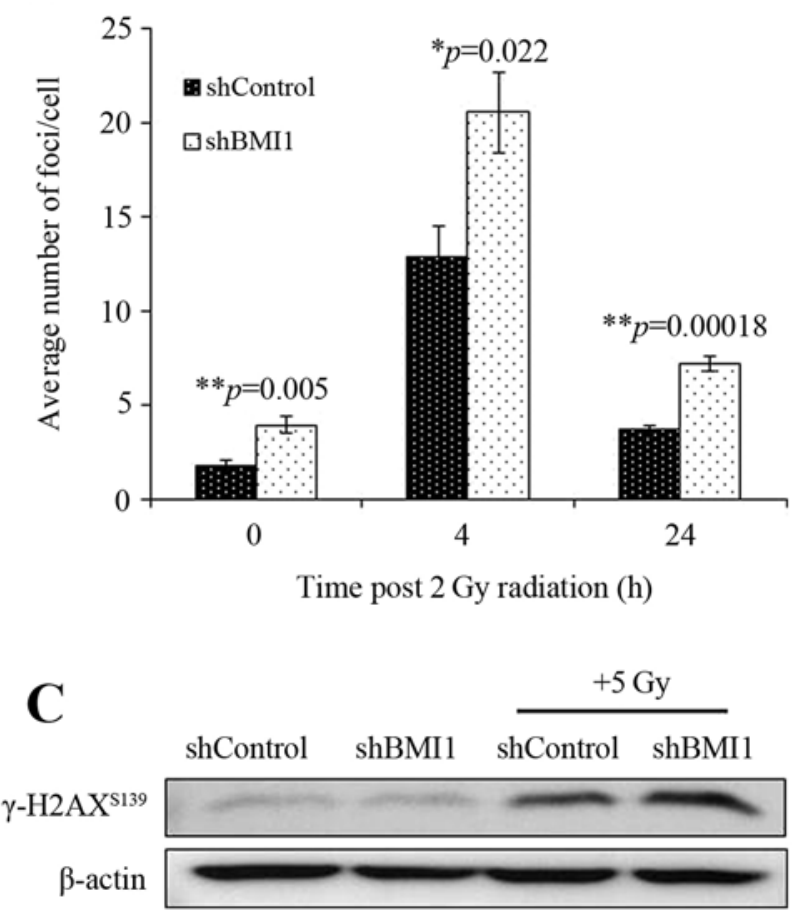

Figure 3. Silencing BMI1 induces DNA damage in MDA-MB-231 cells. Sub-confluent MDA-MB-231 shControl or shBMI1 cells were grown on coverslips for $24 \mathrm{~h}$, exposed to $2 \mathrm{~Gy}$ dose of radiation, incubated for various times after irradiation and stained for $\gamma$-H2AX. (A) Representative photomicrographs of MDA-MB-231 from various treatment conditions and time points are shown. Green stain: $\gamma$-H2AX foci. (B) $\gamma$-H2AX foci were quantified and plotted as the number of foci per nucleus. Mean \pm SE number of foci per nucleus are shown. " $\mathrm{p} \leq 0.05,{ }^{* *} \mathrm{p}<0.005$. (C) Western blotting for $\gamma$-H2AX showed increased $\gamma-\mathrm{H} 2 \mathrm{AX}$ expression in shBMI1 plus radiation treated MDA-MB-231 cells compared to controls. $\beta$-actin was used as a loading control.

$\mathbf{A}$

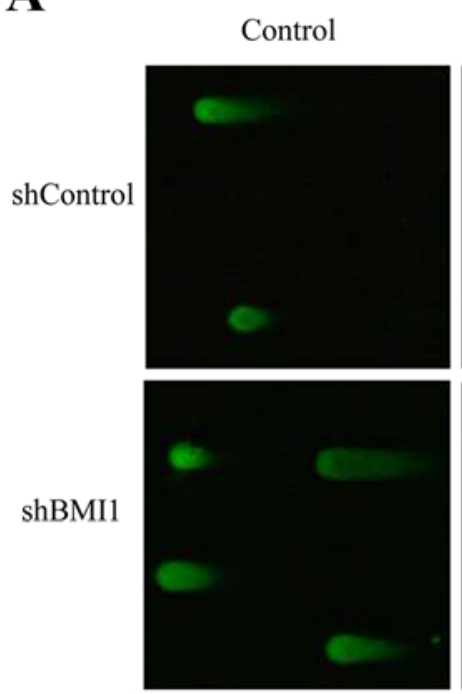

MDA-MB-231

$24 \mathrm{~h}$
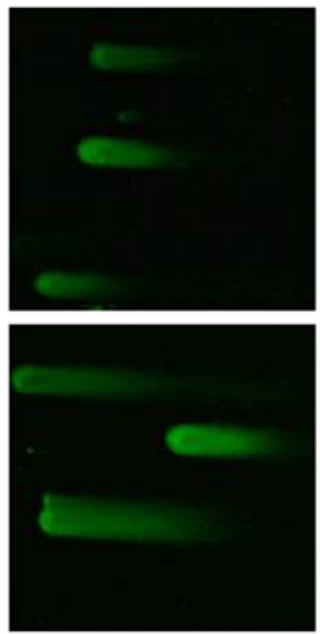

B

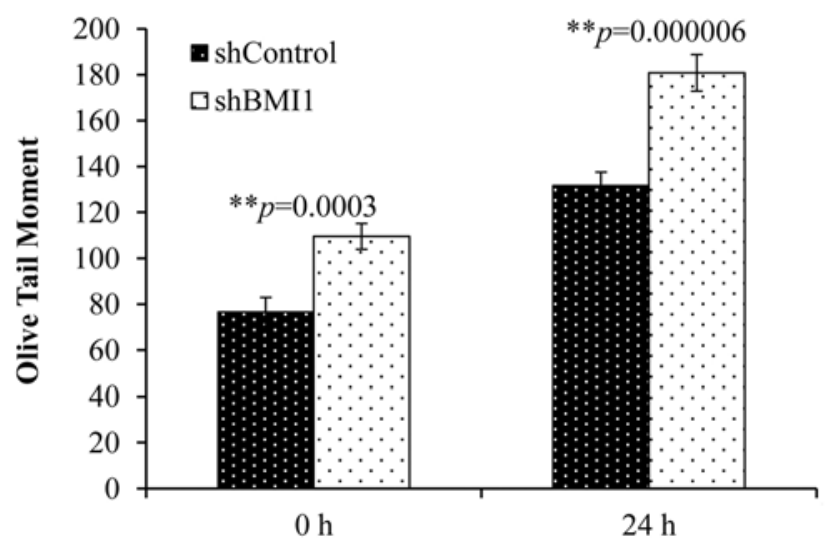

Figure 4. Targeting BMI1 causes DNA damage. (A) Representative Comet images of DSBs detected by neutral Comet assay demonstrate the kinetics of tail moment in shControl or shBMI1 cells without radiation or $24 \mathrm{~h}$ post-irradiation. (B) Increased comet tail moment was observed in shBMI1 cells compared to shControl at 0 and $24 \mathrm{~h}$ post-irradiation. Olive tail moment $(\mathrm{OTM})$ values were determined following the algorithm $($ Olive tail moment $=$ tail mean - head mean) tail \% DNA/100) using Casplab software. Error bars represent standard error of the mean. ${ }^{* *} \mathrm{p} \leq 0.005$.

and modulated repair processes observed in the neutral Comet assay and $\gamma-\mathrm{H} 2 \mathrm{AX}$ foci data, we examined the DNA damage response and repair proteins as supportive evidence. Immunoblot analysis showed that BMI1 silencing reduced the levels of ATM which were further reduced in combination with radiation in both the cell lines (Fig. 5A and B). The expression profiles of DNA-PK, Ku70 and Ku80, proteins involved in DNA repair, were also markedly reduced upon BMI1 inhibition and radiation in both MDA-MB-231 cells and the SUM159PT cells (Fig. 5A and B). However, the reduction in the expression of the individual proteins varied between the two cell lines. Expression of MRE11 was higher in shBMI1 




MDA-MB-231
B
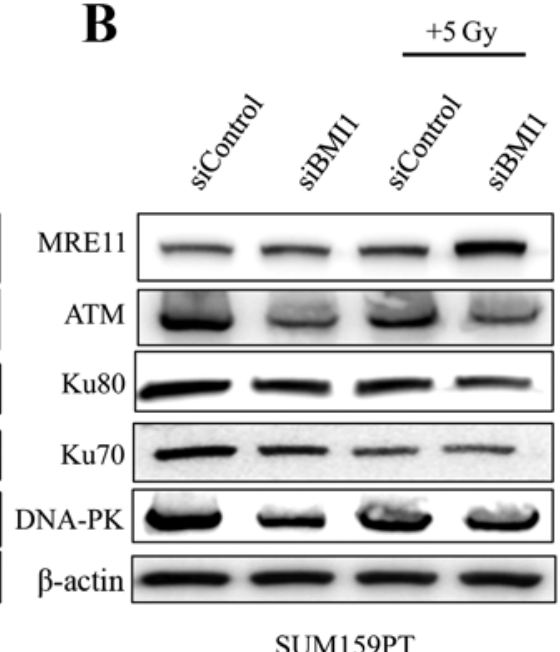

SUM159PT
C
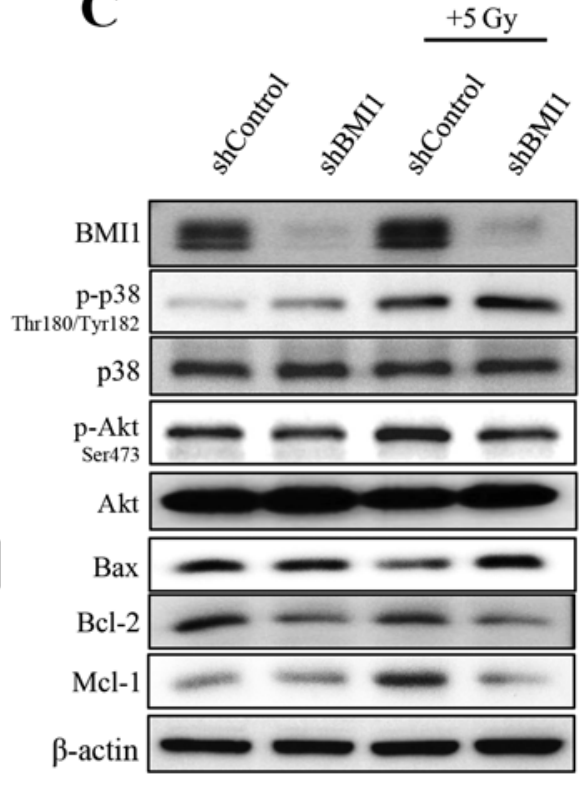

MDA-MB-231

Figure 5. BMI1 modulates apoptotic and DNA damage response protein expression. (A and C) MDA-MB-231 (shControl and shBMI1) and (B) SUM159PT (siScr and siBMI1) cells were grown for $24 \mathrm{~h}$ and treated with or without $5 \mathrm{~Gy}$ radiation. Two hours post-radiation, cell lysates were evaluated by western blotting using the respective antibodies. $\beta$-actin was used as a loading control.

cells treated with radiation, indicating that the maintenance of DNA damage signaling is likely perturbed.

In addition to increased DNA damage, shBMI1 cells demonstrated a marked decrease in the expression profiles of anti-apoptotic proteins $\mathrm{Mcl} 1$ and $\mathrm{Bcl} 2$, and an increase in pro-apoptotic Bax when combined with radiation (Fig. 5C). The regulation of apoptotic proteins can occur through two main pathways: the Akt pathway, which promotes survival by positively regulating the expression of anti-apoptotic proteins; and the MAPK pathway, which leads to the activation of pro-apoptotic proteins. To evaluate the effect of BMI1 downregulation on these pathways, we analyzed the phosphorylation levels of Akt and p38MAPK. We found that knockdown of BMI1 alone increased the activity of pp38Thr180/Tyr182 and these levels were further increased after radiation (Fig. 5C). In addition, pAktSer473 levels decreased with BMI1 knockdown and decreased further after exposure to 5 Gy radiation. These results suggest that perturbation of DNA damage signaling due to impaired activation of ATM in shBMI1 cells leads to accumulation of DNA damage and activation of the p38 MAPK pathway, which in turn modulates the expression of proteins of the apoptotic machinery. Concomitantly, reduced pAKT, Bcl-2 and Mcl1 protein expression was observed in shBMI1 plus radiation.

BMII influences autophagy. Autophagy is typically associated with survival after genotoxic stress (21). Our results showed activation of the autophagic processes in shBMI1 and siBMI1 treated cells without radiation, as indicated by an increase in LC3B-II (Fig. 6A and B). Twenty-four hours after radiation, we observed further enhancement in LC3B-II expression, continuing through $48 \mathrm{~h}$ in the shBMI1 and the siBMI1 knockdown cells, indicating BMI1's regulatory role in autophagy. The shControl cells began to show increase in LC3B-II above the basal level only after radiation treatment followed by $48 \mathrm{~h}$ incubation (Fig. 6A and B). SQSTM1 (also known as p62) is known to associate with LC3 and facilitate the delivery of ubiquitinated proteins to autophagic complexes. As autophagy progresses, p62 and targeted proteins are degraded. BMI1 knockdown cells were observed to have lower basal expression of p62 than shControl cells. This expression further decreases over time after radiation in the shBMI1 as well as the siBMI1 treated cells (Fig. 6A and B). We also carried out qRT-PCR to examine the expression of autophagy-related genes (ATG) in shBMI1 cells, and observed marked upregulation in ATG3, ATG5, ATG7, ATG12, LC3A, and LC3B mRNAs when BMI1 was inhibited, further supporting our findings that genetic inhibition of BMI1 makes breast cancer cells more prone to cell death by autophagy (Fig. 6C).

\section{Discussion}

BMI1 is an important regulator of cancer stem cell (CSC) phenotype and is often overexpressed in cancer cells leading to resistance to therapy $(10,16,22)$. While several studies have shown that BMI1 inhibition increased susceptibility to cytotoxic agents and radiotherapy, the role of BMI1 in radiation response, especially in breast cancer, remains unknown. A number of mechanisms could account for this resistance, including the tumor microenvironment and altered expression of DDR and DNA repair pathway components. In order to explore the mechanisms responsible for radiation resistance in breast cancer, we first showed that silencing of BMI1 increased the sensitivity of MDA-MB-231 and SUM159PT cells to radiation in vitro. While our clonogenic survival data showed that silencing BMI1 resulted in radiosensitization, the underlying mechanism is still unknown. Ionizing radiation mainly exerts its therapeutic effect by causing DNA-DSBs, which are largely 
A

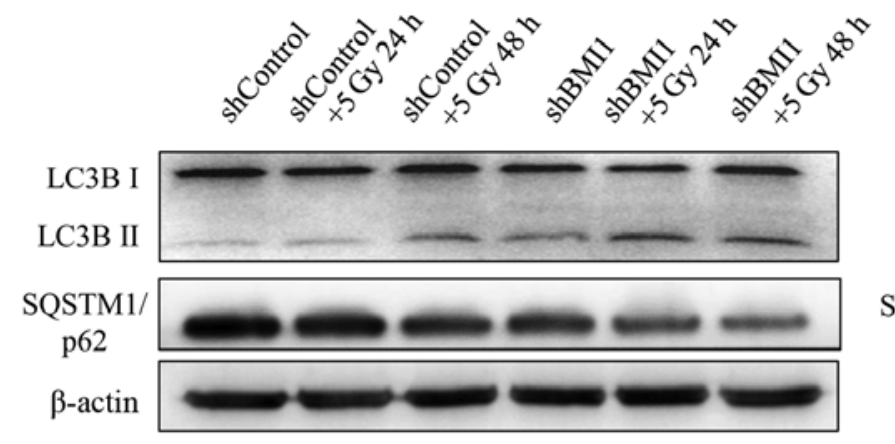

B
SUM159PT

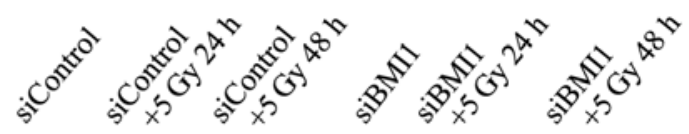

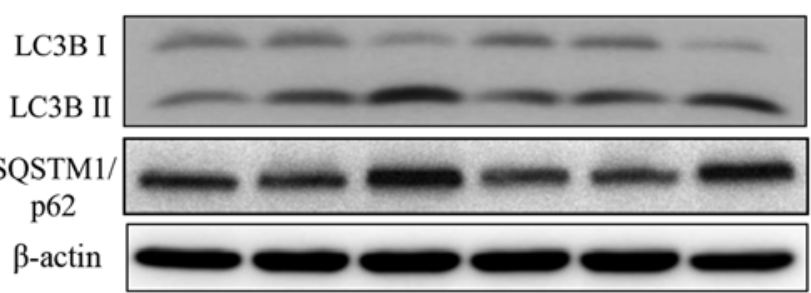
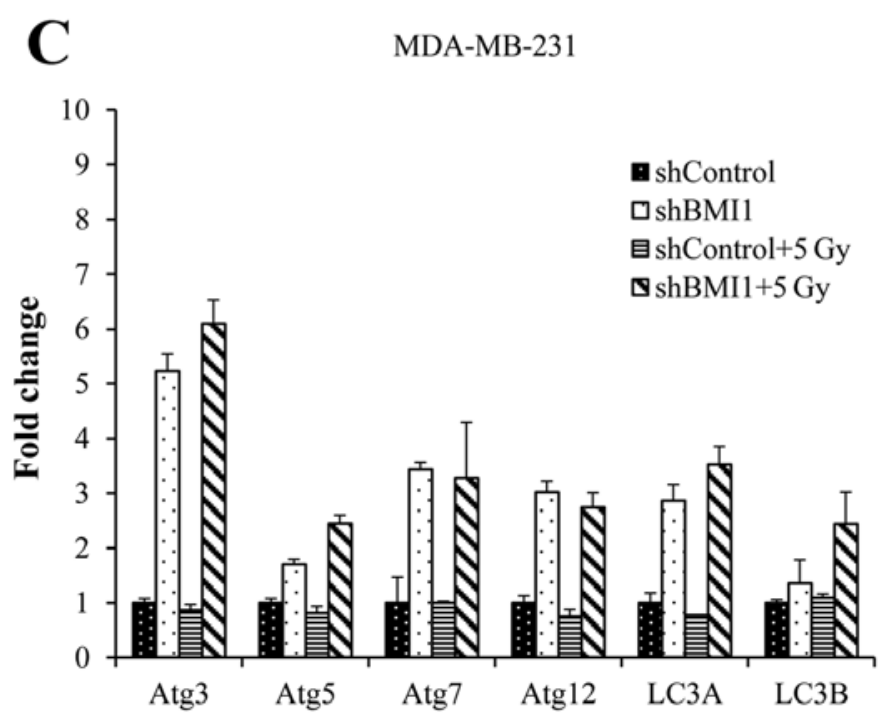

Figure 6. BMI1 influences autophagy. Immunoblot analysis of autophagy proteins in (A) MDA-MB-231 shControl and shBMI1; (B) SUM159PT siScr and siBMI1 cells grown for $24 \mathrm{~h}$ and treated with or without $5 \mathrm{~Gy}$ radiation for 24 and $48 \mathrm{~h}$. $\beta$-actin was used as a loading control. (C) qRT-PCR analysis for autophagy markers in MDA-MB-231 shControl or shBMI1 cells grown for $24 \mathrm{~h}$ and treated with or without 5 Gy radiation for $2 \mathrm{~h}$, normalized to GAPDH. Results showed an increase in autophagy markers in shBMI1 and shBMI1 plus radiation treated cells compared to controls.

responsible for most ionizing radiation-induced cellular lethality $(23,24)$. Hence, the ability of cells to recognize and respond to DSBs is fundamental in determining the sensitivity (or resistance) of cells to radiation. The non-homologous endjoining (NHEJ) pathway is an important pathway for repairing radiation-induced DSBs (23-25). Loss or mutations in any of its subunits (DNA-PK, Ku70 or Ku80) can lead to extreme radiosensitivity and DSB repair deficiency (23). We examined the expression levels of DNA-PK, Ku70, and Ku80, and found them to be markedly reduced in BMI1 silenced cells after irradiation (Fig. 5A and B). We also assessed the levels of ATM, key protein in the (HR) pathway in eukaryotes. BMI1 inhibition suppressed ATM levels upon combination with radiation, indicating that inhibition of the NHEJ and HR pathway, when targeting BMI1, could be a mechanism underlying the observed radiosensitization.

Since BMI1 knockdown produced a reduction in the DNA repair proteins, we hypothesized that the radiosensitizing effect is due to a delay in the repair of radiation-induced DSBs with involvement of the DDR pathway. Several published works have used the phosphorylation of $\mathrm{H} 2 \mathrm{AX}(\gamma-\mathrm{H} 2 \mathrm{AX})$ and its clearance after damage as a measure of radioresistance $(19,20)$. To evaluate a potential delay in DNA damage repair, we assessed $\gamma-\mathrm{H} 2 \mathrm{AX}$ levels and found that BMI1 inhibition greatly impairs the ability of MDA-MB-231 cells to repair radiation-induced DNA damage as observed by the persistent levels of $\gamma-\mathrm{H} 2 \mathrm{AX}$ in shBMI1 cells (Fig. 3). As an additional measure of the effects of shBMI1 on radiation-induced DSBs, we used the Comet assay, which under neutral $\mathrm{pH}$ conditions selectively detects DSBs over single-strand breaks. Our data revealed an increased magnitude of DNA damage in BMI knockdown cells compared with control cells (Fig. 4). These results provide additional evidence of defective DSB repair and offer a partial mechanism for the radiosensitization observed in clonogenic cell survival assays.

Several researchers have evaluated the relationship between BMI1, mitochondrial function, and programmed cell death (PCD) (26). Though the links between type 1 PCD (apoptosis) and type 2 PCD (autophagy) are well established, the question of whether autophagy occurs as a final survival attempt or directly results in cell death is a point of contention for many experts. For some cancers, the induction of autophagy results in radioresistance through enhanced clearance of damaged organelles and protection from cytosolic acidification (27). 
Induction of autophagy through downregulation of Akt/mTOR sensitizes other cancers to radiation therapy (28). Of note, p38 MAPK also appears to play a role in the switch between autophagy and apoptosis, and is capable of acting as both a positive and negative regulator of autophagy (29). A recent study of T-cell acute lymphoblastic leukemia cells showed that resveratrol decreased Akt activation with significant upregulation of autophagy-related genes in a p38-dependent manner (30). In our study shBMI1 cells displayed increased phosphorylation of pp38Thr180/Tyr182, decreased levels of pAktSer473, and significant evidence supporting increased autophagic activity (Figs. 5 and 6). We found that BMI1 knockdown induced autophagy that was greatly exacerbated upon radiation as shown by increased cleavage of LC3B-I to LC3B-II and degradation of p62 (Fig. 6A). Additional evidence comes from our qRT-PCR analysis that shows a significant upregulation of the autophagy genes ATG3, ATG5, ATG7, and ATG12 (Fig. 6C), further supporting the involvement of autophagy in BMI1mediated radiosensitization.

Altogether, our data present evidence for BMI1 as an important target for breast cancer therapy and offers a strategy for incorporating BMI1 targeted therapy with radiation for achieving enhanced radiosensitivity. Histone deacetylase inhibitors, trichostatin A and sodium butyrate, have been shown to reverse acquired radioresistance in esophageal carcinoma cells through downregulation of BMI1 (31). Similarly, the anti-malarial drug, artemisinin, inhibits BMI1 at the protein and transcript levels and radiosensitizes human cervical cancer cells to ionizing radiation $(32,33)$. While these studies have demonstrated BMI1 inhibition produces radiosensitization the drugs tested are not specific BMI1 inhibitors. However, the availability of a novel small-molecule inhibitor of BMI1, PTC-209, provides an opportunity to investigate the effects of BMI1 function in various cancers, including breast. The antitumor effects of PTC-209 have been demonstrated in colorectal cancer, myeloid leukemia and biliary cancer (34-36). In particular, PTC-209 has been shown to target chemotherapy resistant cancer stem cell populations by reducing the function, activity and amount of BMI1 (34).

Up to now, no data is available describing the effects of PTC-209 on breast cancer. As more than 50\% of breast cancer patients receive radiotherapy during the treatment of their disease and breast cancer stem cells are known to be resistant to ionizing radiation, compounds targeting breast cancer stem cells may be better therapeutic agents for treating breast cancer and controlling recurrence and metastasis. Therefore, evaluating PTC-209 as a potential therapeutic agent to restore the sensitivity of breast cancer cells to radiation therapy will be of clinical significance.

\section{Acknowledgements}

This study was supported in part by grants received from an Institutional Development Award (IDeA) from the National Institute of General Medical Sciences (P20 GM103639) of the National Institutes of Health (NIH), a National Cancer Institute (NCI) grant R01 CA167516 received from the NIH, the Stephenson Cancer Center Seed Grant, and by startup funds received from the Department of Radiation Oncology, The University of Oklahoma Health Sciences Center. We thank the Stephenson Cancer Center at the University of Oklahoma Health Sciences Center, Oklahoma City, OK for the use of the Functional Genomics Core which provided molecular analysis service. R.R. is an Oklahoma TSET Research Scholar and holds the Jim and Christy Everest Endowed Chair in Cancer Developmental Therapeutics.

\section{References}

1. Simon JA and Kingston RE: Mechanisms of polycomb gene silencing: Knowns and unknowns. Nat Rev Mol Cell Biol 10: 697-708, 2009.

2. Wang W, Qin JJ, Voruganti S, Nag S, Zhou J and Zhang R: Polycomb group $(\mathrm{PcG})$ proteins and human cancers: Multifaceted functions and therapeutic implications. Med Res Rev 35: 1220-1267, 2015.

3. Park IK, Qian D, Kiel M, Becker MW, Pihalja M, Weissman IL, Morrison SJ and Clarke MF: Bmi-1 is required for maintenance of adult self-renewing haematopoietic stem cells. Nature 423: 302-305, 2003

4. Bruggeman SW, Valk-Lingbeek ME, van der Stoop PP, Jacobs JJ, Kieboom K, Tanger E, Hulsman D, Leung C, Arsenijevic Y, Marino S, et al: Ink4a and Arf differentially affect cell proliferation and neural stem cell self-renewal in Bmil-deficient mice. Genes Dev 19: 1438-1443, 2005.

5. Yang MH, Hsu DS, Wang HW, Wang HJ, Lan HY, Yang WH, Huang CH, Kao SY, Tzeng CH, Tai SK, et al: Bmil is essential in Twist1-induced epithelial-mesenchymal transition. Nat Cell Biol 12: 982-992, 2010.

6. Jacobs JJ, Kieboom K, Marino S, DePinho RA and van Lohuizen M: The oncogene and Polycomb-group gene bmi-1 regulates cell proliferation and senescence through the ink4a locus. Nature 397: 164-168, 1999.

7. Zhang FB, Sui LH and Xin T: Correlation of Bmi-1 expression and telomerase activity in human ovarian cancer. Br J Biomed Sci 65: 172-177, 2008.

8. Vonlanthen S, Heighway J, Altermatt HJ, Gugger M, Kappeler A, Borner MM, van Lohuizen M and Betticher DC: The bmi-1 oncoprotein is differentially expressed in non-small cell lung cancer and correlates with INK4A-ARF locus expression. Br J Cancer 84: 1372-1376, 2001.

9. Kim JH, Yoon SY, Kim CN, Joo JH, Moon SK, Choe IS, Choe YK and Kim JW: The Bmi-1 oncoprotein is overexpressed in human colorectal cancer and correlates with the reduced $\mathrm{p} 16^{\mathrm{INK} 4 \mathrm{a}} / \mathrm{p} 14^{\mathrm{ARF}}$ proteins. Cancer Lett 203: 217-224, 2004.

10. Kim JH, Yoon SY, Jeong SH, Kim SY, Moon SK, Joo JH, Lee Y, Choe IS and Kim JW: Overexpression of Bmi-1 oncoprotein correlates with axillary lymph node metastases in invasive ductal breast cancer. Breast 13: 383-388, 2004.

11. Silva J, García V, García JM, Peña C, Domínguez G, Díaz R, Lorenzo Y, Hurtado A, Sánchez A and Bonilla F: Circulating Bmi-1 mRNA as a possible prognostic factor for advanced breast cancer patients. Breast Cancer Res 9: R55, 2007.

12. Zhang R, Xu LB, Yue XJ, Yu XH, Wang J and Liu C: Bmil gene silencing inhibits the proliferation and invasiveness of human hepatocellular carcinoma cells and increases their sensitivity to 5-fluorouracil. Oncol Rep 29: 967-974, 2013.

13. Xu XH, Liu XY, Su J, Li DJ, Huang Q, Lu MQ, Yi F, Ren JH and Chen WH: ShRNA targeting Bmi-1 sensitizes CD44+ nasopharyngeal cancer stem-like cells to radiotherapy. Oncol Rep 32: 764-770, 2014.

14. Wang G, Liu L, Sharma S, Liu H, Yang W, Sun X and Dong Q: Bmi-1 confers adaptive radioresistance to KYSE-150R esophageal carcinoma cells. Biochem Biophys Res Commun 425: 309-314, 2012.

15. Dong Q, Oh JE, Chen W, Kim R, Kim RH, Shin KH, McBride WH, Park NH and Kang MK: Radioprotective effects of Bmi-1 involve epigenetic silencing of oxidase genes and enhanced DNA repair in normal human keratinocytes. J Invest Dermatol 131: 1216-1225, 2011.

16. Facchino S, Abdouh M, Chatoo W and Bernier G: BMI1 confers radioresistance to normal and cancerous neural stem cells through recruitment of the DNA damage response machinery. J Neurosci 30: 10096-10111, 2010.

17. Long CL, Berry WL, Zhao Y, Sun XH and Humphrey MB: E proteins regulate osteoclast maturation and survival. J Bone Miner Res 27: 2476-2489, 2012. 
18. Muralidharan R, Panneerselvam J, Chen A, Zhao YD, Munshi A and Ramesh R: HuR-targeted nanotherapy in combination with AMD3100 suppresses CXCR4 expression, cell growth, migration and invasion in lung cancer. Cancer Gene Ther 22: 581-590, 2015.

19. Munshi A, Tanaka T, Hobbs ML, Tucker SL, Richon VM and Meyn RE: Vorinostat, a histone deacetylase inhibitor, enhances the response of human tumor cells to ionizing radiation through prolongation of gamma-H2AX foci. Mol Cancer Ther 5: 1967-1974, 2006.

20. Munshi A, Kurland JF, Nishikawa T, Tanaka T, Hobbs ML, Tucker SL, Ismail S, Stevens C and Meyn RE: Histone deacetylase inhibitors radiosensitize human melanoma cells by suppressing DNA repair activity. Clin Cancer Res 11: 4912-4922, 2005.

21. Kroemer G, Mariño G and Levine B: Autophagy and the integrated stress response. Mol Cell 40: 280-293, 2010.

22. Proctor E, Waghray M, Lee CJ, Heidt DG, Yalamanchili M, Li C, Bednar F and Simeone DM: Bmil enhances tumorigenicity and cancer stem cell function in pancreatic adenocarcinoma. PLoS One 8: e55820, 2013.

23. Santivasi WL and Xia F: Ionizing radiation-induced DNA damage, response, and repair. Antioxid Redox Signal 21: 251-259, 2014.

24. Khanna KK and Jackson SP: DNA double-strand breaks: Signaling, repair and the cancer connection. Nat Genet 27 247-254, 2001.

25. Olive PL: The role of DNA single- and double-strand breaks in cell killing by ionizing radiation. Radiat Res 150 (Suppl): S42-S51, 1998

26. Liu J, Cao L, Chen J, Song S, Lee IH, Quijano C, Liu H, Keyvanfar K, Chen H, Cao LY, et al: Bmil regulates mitochondrial function and the DNA damage response pathway. Nature 459: 387-392, 2009

27. Apel A, Herr I, Schwarz H, Rodemann HP and Mayer A: Blocked autophagy sensitizes resistant carcinoma cells to radiation therapy. Cancer Res 68: 1485-1494, 2008.
28. Fujiwara K, Iwado E, Mills GB, Sawaya R, Kondo S and Kondo Y: Akt inhibitor shows anticancer and radiosensitizing effects in malignant glioma cells by inducing autophagy. Int J Oncol 31: 753-760, 2007.

29. Sui X, Kong N, Ye L, Han W, Zhou J, Zhang Q, He C and Pan H: p38 and JNK MAPK pathways control the balance of apoptosis and autophagy in response to chemotherapeutic agents. Cancer Lett 344: 174-179, 2014

30. Ge J, Liu Y, Li Q, Guo X, Gu L, Ma ZG and Zhu YP: Resveratrol induces apoptosis and autophagy in T-cell acute lymphoblastic leukemia cells by inhibiting Akt/mTOR and activating p38-MAPK. Biomed Environ Sci 26: 902-911, 2013.

31. Dong Q, Sharma S, Liu H, Chen L, Gu B, Sun X and Wang G: HDAC inhibitors reverse acquired radio resistance of KYSE-150R esophageal carcinoma cells by modulating Bmi-1 expression. Toxicol Lett 224: 121-129, 2014.

32. Gong XM, Zhang Q, Torossian A, Cao JP and Fu S: Selective radiosensitization of human cervical cancer cells and normal cells by artemisinin through the abrogation of radiation-induced G2 block. Int J Gynecol Cancer 22: 718-724, 2012.

33. Wu J, Hu D, Yang G, Zhou J, Yang C, Gao Y and Zhu Z: Down-regulation of BMI-1 cooperates with artemisinin on growth inhibition of nasopharyngeal carcinoma cells. J Cell Biochem 112: 1938-1948, 2011.

34. Kreso A, van Galen P, Pedley NM, Lima-Fernandes E, Frelin C Davis T, Cao L, Baiazitov R, Du W, Sydorenko N, et al: Selfrenewal as a therapeutic target in human colorectal cancer. Nat Med 20: 29-36, 2014.

35. Bolomsky A, Schlangen K, Schreiner W, Zojer N and Ludwig H: Targeting of BMI-1 with PTC-209 shows potent anti-myeloma activity and impairs the tumour microenvironment. J Hematol Oncol 9: 17, 2016.

36. Mayr C, Wagner A, Loeffelberger M, Bruckner D, Jakab M, Berr F, Di Fazio P, Ocker M, Neureiter D, Pichler M, et al: The BMI1 inhibitor PTC-209 is a potential compound to halt cellular growth in biliary tract cancer cells. Oncotarget 7: 745-758, 2016. 